\title{
Research International Journal of Anesthesiology
}

\section{Short Communication}

\section{Stroke Associated Pneumonia Impact and Causality}

\section{Nilanchal Chakraborty*}

Department of Anesthesiology and Critical Care Medicine, Institute of Neurosciences Kolkata.

*Address for Correspondence: Nilanchal Chakraborty, MD, Department of Anesthesiology and Critical Care Medicine, Institute of Neurosciences Kolkata. E-mail: nilanchal83@gmail.com

Received: 08 May 2020; Accepted: 01 June 2020; Published: 02 June 2020

Citation of this article: Chakraborty N (2020) Stroke Associated Pneumonia Impact and Causality. Re Int Jou of Anes. 1(1): $001-002$. DOI: 10.37179/rija.000001.

Copyright: (C) 2020 Chakraborty N. This is an open access article distributed under the Creative Commons Attribution License, which permits unrestricted use, distribution, and reproduction in any medium, provided the original work is properly cited.

\section{Short Communication}

Medical complications like pneumonia, urinary tract infections, or deep venous thrombosis, occurs in almost one third of patients of stroke [1]. Stroke associated pneumonia (SAP) is the terminology for the spectrum of pneumonia complicating the first 7 days after stroke in non-ventilated patients which is one of the most frequent of these complications affecting 2.3 to $28 \%$ of patients with stroke.

Stroke associated dysphagia and possible aspiration is not the only cause of pneumonia in these cases.

Stroke induces a rapid and temporary immunosuppression mainly mediated by autonomic nervous system. [2] Stroke induced immunosuppression syndrome (SIDS) causes rapid functional deactivation of monocytes and numerical decrease in peripheral blood lymphocytes resulting in increased susceptibility for stroke associated pneumonia. It also suppresses innate immunity and neutrophil function, drastic loss of circulating, splenic and thymic $\mathrm{T}$ cells [3].

Stroke induced innate immunosuppression can persist for months and years following ischemia [4]. Larger stroke size, or lesion localised to middle or superior temporal gyri may be associated with greater immunosuppression [5]. Some studies have shown that not only the presence dysphagia, but lower mHLA-DR (monocyte HLADR) expression or impaired monocyte function, higher IL-6 and LBP expression on day 1 of stroke were significantly associated with SAP $[2]$.

Some other studies have shown that SAP was significantly associated with older age, higher initial NIHSS (National Institute of Health Stroke Scale) score, dysphagia on admission and history of atrial fibrillation and led to more frequent mechanical ventilation and death [1]. Chest radiography is frequently normal in the early evaluation of both communities acquired pneumonia (CAP) or hospital acquired pneumonia (HAP), similarly in suspected SAP, typical diagnostic appearances on initial chest $\mathrm{x}$-ray were present in only $36 \%$ of patients [6].

Cultures in SAP often remain negative without bacterial growth. Some studies have used procalcitonin level of $>0.05 \mathrm{ng} / \mathrm{ml}$ as a predictive biomarker of possible infection and they treated patients with antibiotic to prevent stroke associated pneumonia. There was no difference in infection rate or stroke related outcome as per modified Rankin scale in antibiotic treated or placebo group in this study [7].

In another study, prophylactic antibiotic therapy did not reduce incidences of post stroke pneumonia, and all-cause mortality was also same. Although it reduced incidences of catheter associated urinary tract infections but increased chances of Clostridium difficile associated diarrhoea, and increased methicillin-resistant staphylococcus aureus colonisation. Prophylactic antibiotic also increased length of hospital stay and poor outcome at 90 days in these patients [8].

Novel immunomodulatory methods like beta blockers or gut microbiota are still in experimental stage to address the stroke associated complex immunosuppression [9]. Organisms isolated from sputum or tracheal aspirate of stroke patients are commonly the organisms associated with HAP (Hospital Acquired Pneumonia) like Pseudomonas sp, Klebsiella sp, Enterobacter sp, E. coli compared to organisms associated with Community acquired Pneumonia (CAP) even within 48 hours of admission.

Hence prompt presumption and diagnosis of any infection 
is very much necessary. Early initiation of antibiotics as per local microbiological flora followed by prompt culture-based modification is also needed to manage stroke associated infections, especially stroke associated pneumonia. Prompt and individualised clinical judgement and judicious application of antibiotics for treatment of infections can only improve outcomes in stroke. Beta blockers or other immunomodulatory agents are only there for future experiments and a long way to go before they come in meaningful clinical practice.

\section{References}

1. Emmanuelle C, Igor S (2017) Stroke- Associated Pneumonia Risk score: Validity in French stroke unit: Journal of stroke and Cerebrovascular diseases 26: 225-229. Link: https://bit.ly/3eo1iQ1

2. Sarah H, Hendrik H, Lena U, Darius NG, Bruno-MM, et all. (2017) Stroke- induced immunodepression and dysphagia independently predict stroke- associated pneumonia- The PREDICT study 37: 36713682. Link: https://bit.ly/2ZEshTe

3. Offner H, Sandhya S, Susan MP, Chunhe W, Michael EA, et al. (2006) Splenic atrophy in experimental stroke is accompanied by increased regulatory T cells and circulating macrophages. Journal of Immunology 176: 6523-6531. Link: https://bit.ly/3emC44n

4. Theodorou G, Marousi S, Ellul J, Mougiou A, Theodori E, eta al. (2008) T helper 1 (Th1)/Th2 cytokine expression shift of peripheral blood
CD4+ and CD8+ T cells in patients at the post-acute phase of stroke. Clinical and Experimental Immunology 152: 456-463. Link: https:// bit.ly/2XxYl8H

5. Urra X, Carlos L, Yashu Z, Sergio A, Salvatore R, et al. (2017) Neuroanatomical correlates of stroke-associated infection and strokeinduced immunodepression. Brain, Behavior, and Immunity 60: 142150. Link: https://bit.ly/2TKi3gt

6. Smith CJ, Amit KK, Andy V, Angel C, Javier G, et al. (2015) Diagnosis of Stroke-Associated Pneumonia: Recommendations from the Pneumonia in Stroke Consensus Group. Stroke. 46: 2335-2340. Link: Link: https://bit.ly/2ZRA9kD

7. Lena Ulm, Sarah H, Darius N, Marcella H, Bruno-MM, et al. (2017) The Randomized controlled STRAWINSKI trial: Procalcitonin- guided antibiotic therapy after stroke. Frontiers in Neurology 8: 153. Link: https://bit.ly/2X93k0N

8. Lalit K, Saddif I, John H, Matthew S, Martin G, et al. (2015) Prophylactic antibiotics after acute stroke for reducing pneumonia in patients with dysphagia (STROKE-INF): a prospective, cluster-randomised, openlabel, masked endpoint, controlled clinical trial. The Lancet 386: 1835-1844. Link: https://bit.ly/2Xy0npl

9. Ilko L, André K, Rafael M, Mathias B, Jan Liman (2015) Effect of BetaBlocker Therapy on the Risk of Infections and Death after Acute Stroke - A Historical Cohort Study. PLOs One 2: 10. Link: https://bit. ly/3c6ve0Q 